\title{
Efeito agudo da automassagem e alongamento passivo de membros inferiores no
}

\section{desempenho do salto vertical}

\author{
Acute effect of lower limbs self-massage and passive stretching on vertical jump performance \\ Efecto agudo del automasaje y estiramiento pasivo de miembros inferiores sobre el rendimiento del
} santo vertical

\section{Resumo}

O objetivo do presente estudo foi verificar o efeito agudo do alongamento estático e da automassagem com foam rolling nos membros inferiores sobre o desempenho de salto vertical. Fizeram parte do presente estudo 10 participantes do sexo feminino fisicamente ativas e aparentemente saudáveis. As participantes realizaram dois protocolos com entrada aleatória, conduzidos pela técnica do quadrado latino, sendo eles: 1) condição controle (CC); 2) automassagem com foam rolling (FR) e 3) alongamento estático (AE). O Sargent Jump Test foi utilizado para testar a potência de membros inferiores, sendo realizado após as condições FR e AE. Ambas as condições FR e AE foram realizadas paras as regiões anterior e posterior da coxa e posterior da perna em 4 séries com 30 segundos de duração para cada posição, respeitando um intervalo passivo de 30 segundos entre cada série. No que diz respeito às condições experimentais, tanto a condição $\mathrm{AE}(\mathrm{p}=0,010)$ quanto a condição $\mathrm{FR}(\mathrm{p}=0,042)$ aumentaram significativamente a distância alcançada nos saltos em relação a condição CC. Não foram observadas diferenças significativas entre as condições experimentais (AE vs FR) testadas ( $\mathrm{p}=1,000)$. De acordo com os resultados do presente estudo, estratégias pré exercício como FR e AE parecem melhorar a potência de membros inferiores no Sargent Jump Test.

Palavras-chave: Dor musculoesquelética; Fadiga muscular; Fáscia; Manipulações musculoesqueléticas. 


\begin{abstract}
The purpose of the present study was to verify the acute effect of static stretching and foam rolling self-massage on the lower limbs on vertical jump performance. Ten physically active and apparently healthy women participated in the present study. Participants performed two protocols with random entry, conducted using the Latin square technique: 1) control condition (CC), 2) self-massage via foam rolling (FR), and 3) static stretching (SS). The Sargent Jump Test was used to assess the lower limbs muscle power being performed before the FR and SS conditions. Both FR and SS conditions were performed for the anterior and posterior thigh and posterior leg regions in 4 sets with 30 seconds duration for each position, respecting a passive recovery of 30 seconds between each set. Both SS $(p=0.010)$ and FR condition $(p=0.042)$ significantly increased the distance reached in jumps in comparison to CC. No statistical difference was observed between experimental conditions (FR vs SS) $(p=1.000)$. According to the present study results both pre-exercise strategies (FR and SS) seem to improve the lower limbs muscle power in the Sargent Jump Test.
\end{abstract}

Keywords: Musculoskeletal pain; Muscle fatigue; Fascia; Musculoskeletal manipulations.

\begin{abstract}
Resumen
El objetivo del presente estudio fue verificar el efecto agudo del estiramiento estático y el automasaje con laminado de espuma en los miembros inferiores sobre la ejecución del salto vertical. Diez participantes femeninas físicamente activas y aparentemente sanas participaron en el presente estudio. Los participantes realizaron dos protocolos con entrada aleatoria, realizados mediante la técnica del cuadrado latino, a saber: 1) condición de control (CC); 2) automasaje con laminado de espuma (LM) y 3) estiramiento estático (EE). Se utilizó el Sargent Jump Test para probar la potencia de los miembros inferiores, que se realizó después de las condiciones de LM y EE. Las condiciones de LM y EE se realizaron para la región anterior y posterior del muslo y la parte posterior de la pierna en 4 series con 30 segundos de duración para cada posición, respetando un intervalo pasivo de 30 segundos entre cada serie. En cuanto a las condiciones experimentales, tanto la condición EE ( $p=0.010)$ como la condición LM ( $p$ $=0.042$ ) incrementaron significativamente la distancia alcanzada en saltos en relación a la condición CC. No hubo diferencias significativas entre las condiciones experimentales $(\mathrm{EE}$ vs $\mathrm{LM})$ probadas $(\mathrm{p}=1.000)$. Según los resultados del presente estudio, las estrategias pre-ejercicio como LM y EE parecen mejorar la potencia de los miembros inferiores en el Sargent Jump Test.
\end{abstract}

Palabras clave: Dolor musculoesquelético; Fatiga muscular; Fascia; Manipulaciones musculoesqueléticas.

\title{
1. Introduçãa
}

Os saltos são considerados como elementos fundamentais e rudimentares para a maioria das atividades desportivas, cuja impulsão e a aplicação de força são determinantes para um bom rendimento (Melin et al., 2019). Os saltos podem ser compreendidos como uma aplicação de força dinâmica (impulsão) com finalidade de movimentar o corpo ou segmento com a maior potência possível se opondo a lei da gravidade pela potência gerada. A potência muscular pode ser considerada um dos aspectos fundamentais para desempenho de atividades físicas e desportivas. Segundo Rodrigues e Marins (2011), os testes de saltos verticais são considerados um dos melhores testes aplicáveis ao campo para avaliar indicadores da potência muscular dos membros inferiores.

Diante dessas consequências se torna necessário compreender as funcionalidades e associar a potência muscular e as características de transmissão de força do sistema miofascial (Myers, 2014). A rede fascial caracteriza-se como um emaranhado de tecido conjuntivo que envolveu todas as nossas estruturas corporais (ex. ossos, vísceras e músculos), sendo considerada como o maior órgão sensorial em extensão do nosso corpo (Schleip, 2003). Anatomicamente, o tecido fascial é formado por duas substâncias estruturais (elastina e colágeno) (Stecco e Schleip, 2016) que, quando necessário, suportam tensões e transmitem forças entre ela (Schleip, 2003).

De forma funcional, a fáscia é extremamente complacente, mas pode sofrer com um forte e contínuo estresse mecânico, o que por consequência perderia a efetividade dessa característica (Behara e Jacobson, 2015; Stecco e Schleip, 2016). Assim, diversas técnicas veem sendo testadas ao longo do tempo com intuito de minimizar tais efeitos. As duas principais técnicas encontradas na literatura são tanto o alongamento quando a automassagem com foam rolling (Monteiro et al., 2017; 2018). 
Atualmente, a automassagem com foam rolling vem ganhando destaque na literatura dada suas contribuições positivas para ganhos de amplitude articular (Monteiro et al., 2017; 2019), mas que ainda indica uma incerteza ao associá-la com movimentos que exijam força muscular (Monteiro e Corrêa Neto, 2016; Monteiro et al., 2017; 2019). Num dos poucos trabalhos encontrados na literatura, Zhang et al. (2020) não foram capazes de observar alterações significativas no desempenho do salto vertical após aplicação de um protocolo de alongamento e outro de automassagem com foam rolling.

Dessa forma, o objetivo do presente estudo foi verificar o efeito agudo do alongamento estático e da automassagem com foam rolling nos membros inferiores sobre o desempenho de salto vertical. Como hipótese, espera-se que ambas as técnicas aplicadas nos membros inferiores promovam melhora no desempenho do salto vertical, tendo a automassagem com foam rolling resultados melhores.

\section{Metodologia}

\subsection{Tipo de estudo}

O estudo em tela é uma pesquisa do tipo transversal com característica quantitativa (Estrela, 2018).

\subsection{Amostra}

A amostra foi composta por 10 participantes do sexo feminino (idade: $32,3 \pm 8,7$ anos; estatura: 1,60 \pm 0,06 metros; massa corporal: 66,4 \pm 14,1 quilogramas; índice de massa corporal: 25,5 \pm 3,7) fisicamente ativos e aparentemente saudáveis. Foram considerados elegíveis ao presente estudo os indivíduos que praticavam exercícios físicos há pelo menos um ano e com frequência mínima de três dias semanais e que fosse experiente nas técnicas de alongamento e automassagem com foam rolling. Foram excluídos os indivíduos que apresentassem qualquer tipo de limitação ou condição médica que inviabilizasse a coleta dos dados, bem como respondessem positivamente o Questionário de Prontidão para Atividade Física. A presente pesquisa possui característica de cross-over com entrada aleatória aos protocolos, conduzidas pela técnica do quadrado latino. As participantes foram divididas em três condições, sendo elas: 1) condição controle (CC); 2) condição de automassagem com foam rolling (FR) e 3) condição de alongamento estático (AE).

Previamente, todos os indivíduos foram esclarecidos quanto aos procedimentos que seriam realizados bem como leram e assinaram o Termo de Consentimento Livre Esclarecido. Em conformidade com as premissas éticas, o estudo foi previamente aprovado pelo Comitê de Ética em Pesquisa da Universidade do Grande Rio (CAEE: 61550916.1.0000.5283) e seguiram as normas da Resolução 466/12 do Conselho Nacional de Saúde.

\subsection{Protocolo Experimental}

Cada participante compareceu ao local dos testes em 4 dias não consecutivos com um intervalo mínimo de 24 horas entre as visitas, sendo orientado que não praticasse exercícios físicos entre as visitas. Todos os participantes participaram de todos os protocolos, caracterizando um estudo cross-over. No primeiro dia cada participante realizou uma familiarização, leram e deram consentimento através da assinatura do TCLE, bem como tiveram seus dados antropométricos coletados. A partir da segunda visita, os participantes foram divididos em três condições, sendo elas: 1) CC; 2) FR e 3) AE. Após a aleatorização (técnica do quadrado latino) de entrada das condições, cada participante realizou as condições do dia e foram avaliados no Sargent Jump Test (SJT) para verificar o efeito da técnica no teste. 


\subsection{Procedimentos}

\section{Exercício de alongamento estático}

Para a condição AE foram aplicadas posições de alongamento para as regiões anterior (ex. quadríceps) e posterior de coxa (ex. isquiotibiais) e para a região posterior da perna (ex. panturrilha), sendo adotados três posicionamentos. Durante o primeiro posicionamento foi realizado o movimento de flexão de joelho em pé, onde o sujeito deveria flexionar o joelho segurando no seu ante pé e realizando o maior grau de movimento, respeitando seu limite máximo. Durante o primeiro posicionamento foi realizado o movimento de flexão de tronco com os joelhos estendidos até atingir o maior grau de movimento. Durante o primeiro posicionamento foi realizado o movimento de dorsiflexão em pé. Para a aplicação de todos os posicionamentos os sujeitos foram levados até uma posição de ligeiro desconforto, como indicado pelo Colégio Americano de Medicina do Esporte (ACSM, 2011). Foram aplicadas 4 séries com 30 segundos de duração para cada posição, respeitando um intervalo passivo de 30 segundos entre cada série.

\section{Exercícios de automassagem com foam rolling}

A condição FR foi realizada com um rolo que possui um núcleo rígido revestido por uma camada de espuma de acetato de etileno (Foam Roller Brasil, Porto Alegre, RS, Brasil), sendo escolhido de forma proposital por ser capaz de produzir maior pressão sobre o tecido mole em comparação aos rolos convencionais (Curran e Colaboradores, 2008). A condição FR foi aplicada nas regiões anterior e posterior de coxa, bem como na região posterior da perna no membro inferior dominante. Na região anterior de coxa a técnica foi realizada unilateralmente em posição pronada, enquanto o membro inferior em contato com o rolo era mantido estendido e relaxado. Na região posterior de coxa e posterior de perna a técnica foi realizada unilateralmente em posição sentada, enquanto o membro inferior em contato com o rolo era mantido estendido e relaxado. Os participantes receberam instrução de usar tanto o membro inferior não dominante quanto os membros superiores para rolarem dinamicamente e longitudinalmente entre o acetábulo e o tendão patelar, entre a tuberosidade isquiática e a fossa poplítea e entre o tendão do calcâneo e a fossa poplítea para as regiões anterior e posterior de coxa e posterior de perna, respectivamente. Sempre que possível, os participantes foram encorajados a imprimir a maior pressão sobre o tecido alvo. Para melhor representação do cenário real de treinamento, os participantes ficaram livres para escolherem a cadência de rolamento durante a execução da técnica. Foram aplicadas 4 séries com 30 segundos de duração para cada posição, respeitando um intervalo passivo de 30 segundos entre cada série.

\section{Sargent Jump Test}

Cada participante realizou o SJT sem contramovimento em 3 tentativas, sendo anotado o maior salto entre elas para utilização das comparações. Durante o SJT, os participantes foram instruídos a se posicionar lateralmente à parede com o braço estendido acima da cabeça, o mais alto possível e foi medida a estatura total. Após sujou-se as pontas do dedo médio com tinta guache azul e o participante afastou-se ligeiramente da parede, no sentido lateral para realizar uma série de 3 saltos, mantendo o braço elevado e estendido verticalmente preservando um intervalo de 1 a 3 minutos entre cada salto.

\subsection{Tratamento Estatístico}

Para testar a normalidade dos dados foi aplicado o teste de Shapiro Wilk que rejeitou a normalidade para os dados referentes ao protocolo de automassagem $(\mathrm{p}=0,02)$. Sendo assim, a descrição das medidas foi retratada pela mediana e pelo intervalo interquartílico como medidas de tendência central e de dispersão respectivamente. A comparação entre as condições foi realizada através da estratégia não paramétrica aplicando-se o teste de Friedman, e para pontuar as diferenças foi feita uma 
comparação por pares levando-se em consideração o critério de Bonferroni. Para todo tratamento inferencial foi considerado um nível de significância de $5 \%(\mathrm{p}<0,05)$.

\section{Resultados e Discussão}

No que diz respeito às condições experimentais, tanto a condição $\mathrm{AE}(p=0,010)$ quanto a condição $\mathrm{FR}(\mathrm{p}=0,042)$ aumentaram significativamente a distância alcançada nos saltos em relação a condição CC (Tabela 1). Não foram observadas diferenças significativas entre as condições experimentais (AE vs FR) testadas ( $p=1,000)$.

Tabela 1 - Distância atingida nos saltos para cada condição. Valores representados em mediana e intervalo interquartílico).

\section{PROTOCOLO DISTÂNCIA $(\mathrm{cm})$}

\begin{tabular}{|c|c|}
\hline Saltos sem intervenção & $0,32(0,12)$ \\
\hline Salto com alongamento & $0,36(0,17) *$ \\
\hline Salto com automassagem & $0,37(0,15) *$ \\
\hline
\end{tabular}

O objetivo do presente estudo foi verificar o efeito agudo do alongamento estático e da automassagem com foam rolling nos membros inferiores sobre o desempenho de salto vertical. Os resultados indicaram aumento significativo na distância alcançada nos saltos após as condições AE $(p=0,010)$ e FR ( $p=0,042)$. Dessa forma, a hipótese inicial do presente estudo foi parcialmente confirmada haja visto que foi observado aumento significativo nas condições experimentais (AE e FR), mas não foram observadas diferenças entre as condições.

O principal achado do presente estudo sugere que ambas as técnicas (AE e FR) foram capazes de aumentar significativamente o desempenho no SJT. Este fato vai de encontro com as informações previamente apresentadas pela literatura (Zakas et al., 2006; Toledo et al., 2014). Toledo et al. (2014) observaram efeito deletério no desempenho do salto vertical imediatamente após realizar um protocolo de alongamento estático e jogadores de futebol feminino sub-17, mas com dissipação de efeito já com 10 minutos após a aplicação do protocolo. Anteriormente, Zakas et al. (2006) tinham sugerido que o alongamento estático se mostrava mais efetivo para a ativação de mecanorreceptores responsáveis pelo relaxamento da tensão viscoelástica, musculotendínea, e ativação menor dos reflexos inibitórios gerados pelo órgão tendinoso de golgi, parecendo apresentar efeito negativo em relação a geração de força e potência muscular, o mesmo estudo sugere que há relação direta entre tempo de duração do exercício e sua relação ao volume e a menor ativação e relaxamento muscular.

Este fato parece ter influenciado nas respostas diferentes encontradas no presente estudo, onde o fracionamento do volume total em séries de 30 segundos de duração parece não ter sido suficientes para ativação desses mecanorreceptores 
sugeridos por Zakas et al. (2006). Assim, podemos sugerir que o protocolo utilizado no presente estudo não foi suficiente para produzir alterações mecânicas ou neurais capazes de gerar comprometimento na potência dos membros inferiores. Fato este que parece corroborar com os achados atuais relacionados a técnica de FR e a produção de força. Zhang et al. (2020) realizaram uma série simples de automassagem para a cadeia posterior (região plantar: 60 segundos; panturrilha: 80 segundos; glúteos: 50 segundos; lombares: 50 segundos; músculos suboccipitais: 40 segundos) e não foram capazes de observar diferenças significativas após o protocolo.

Esses resultados vão ao encontro dos achados prévios relacionando o FR com a produção de força. Monteiro e Corrêa Neto (2016) foram os primeiros autores a indicarem aumento no acúmulo de fadiga ao realizar o FR na região anterior de coxa, o que foi posteriormente reforçado por Monteiro et al. (2017) quando observaram redução na produção de força dos extensores de joelho após um volume igual ou superior a 60 segundos de FR realizada na região anterior de coxa. Resultados similares que corroboram posteriormente com essa linha foram publicados por Monteiro et al. (2017; 2019), os quais observaram tanto redução na produção de força dos extensores de joelho quanto aumento no acúmulo de fadiga muscular após um volume igual ou superior a 60 segundos de FR realizada na região anterior de coxa.

Apesar de não testada no presente estudo, alguns mecanismos são propostos na literatura a fim de elucidar as respostas encontradas no presente estudo. $\mathrm{O}$ alongamento, independentemente de passivo/estático ou facilitação neuromuscular proprioceptiva parecem sofre disfacilitação reflexa do fuso muscular, pelo fato da manutenção da posição alongada por um período prolongado, o que por consequência induziria numa redução da frequência de descarga do saco nuclear e da cadeia nuclear (Behm e Chaouachi, 2011; Behm et al., 2016 ${ }^{\mathrm{ab}}$; Behm, 2018; Monteiro e Colaboradores, 2018). Este fato poderia alterar a arquitetura muscular e influenciar negativamente na produção de força, principalmente na potência muscular. Contudo, os resultados do presente estudo não foram capazes de confirmar essa hipótese.

Algumas limitações e delimitações são encontradas na interpretação do presente estudo. Primeiro, a cadência durante cada rolamento na condição FR não foi controlada. Propositalmente, essa possibilidade foi escolhida por melhor representar o cenário real de aplicação dessa técnica e aproximar os resultados de uma maior aplicabilidade prática. Por fim, apesar do investigador estar cego quanto ao protocolo utilizado e a entrada dos participantes terem sido aleatória, é possível que ao decorrer da investigação (sucessão dos protocolos) o fator aprendizado tenha influenciado nas medidas.

\section{Considerações Finais}

De acordo com os resultados do presente estudo, estratégias pré exercício como alongamento estático e automassagem podem resultar em melhora no desempenho de potência. Ainda, existe a necessidade da realização de possíveis estudos observando diferentes volumes e seu impacto na potência muscular, visto que resultados antagônicos advindos de estudos com volumes discrepantes aos aqui descritos são evidenciados na literatura. Assim é real a necessidade de desenvolvimento de novos estudos que caminhem para a manipulação das variáveis de prescrição do FR, principalmente no que tange a interdependência volume-intensidade e o sítio alvo da técnica. A partir dessas elucidações poderá ser melhor compreendido a aplicação da mesma ao longo de exercícios que envolvam trabalho de potência muscular de membros inferiores.

\section{Agradecimentos}

Os autores agradecem a participação de todos os voluntários dessa pesquisa. 


\section{Referências}

Behara, B.; Jacobson, B. H. (2015). The acute effects of deep tissue foam rolling and dynamic stretching on muscular strength, power, and flexibility in division I linemen. J Strength Cond Res, 31 (4), 888-892.

Behm, D. G.; Chaouachi, A. (2011). A review of the acute effects of static and dynamic stretching on performance. Eur J Appl Physiol, 111 (11), $2633-2651$.

Behm, D. G.; Cavanaugh, T.; Quigley, P.; Reid, J. C.; Nardi, P. S.; Marchetti, P. H. (2016a). Acute bouts of upper and lower body static and dynamic stretching increase non-local joint range of motion. Eur J Appl Physiol, 116 (1), 241-249.

Behm, D. G.; Blazevich, A. J.; Kay, A. D.; MacHugh, M. (2016b). Acute effects of muscle stretching on Physical performance, range of motion, and injury incidence in healthy active individuals: a systematic review. Appl Physiol Nutr Metab, 41 (1), 1-11.

Behm, D. G. (2018). The science and physiology of flexibility and stretching: implications and applications in sport performance and health. 1. ed. United Kingdom: Routledge Publishers, pp.139-165.

Curran, P. F.; Fiore, R. D.; Crisco, J. J. (2008). A comparison of the pressure exerted on soft tissue by 2 myofascial rollers. J Sport Rehabil, 17 (4), $432-442$.

Estrela, C. (2018). Metodologia Científica: Ciência, Ensino, Pesquisa. Editora Artes Médicas.

Melin, A. K.; Heikura, I. A.; Tenforde, A.; Mountjoy, M. (2019). Energy availability in athletics: health, performance, and physique. Int J Sport Nutr Exerc Metab, 29 (2), 152-164.

Monteiro, E. R.; Corrêa Neto, V. G. (2016). Effect of different foam rolling volumes on knee extension fatigue. Int J Sports Phys Ther, 11 (7), $1076-1081$.

Monteiro, E. R.; Cavanaugh, M. T.; Frost, D. M.; Novaes, J. S. (2017). Is self-massage an effective joint range-of-motion strategy? A pilot study. J Bodyw Mov Ther, 21 (1), 223-226.

Monteiro, E. R.; Škarabot, J.; Vigotsky, A. D.; Brown, A. F.; Gomes, T. M.; Novaes, J. D. (2017). Maximum repetitions performance after different antagonist foam rolling volumes in the inter-set rest period. Int J Sports Phys Ther, 12 (1), 76-84.

Monteiro, E. R.; Vigotsky, A. D.; Škarabot, J.; Brown, A. F.; Gomes, T. M.; Novaes, J. D. (2017). Acute effects of different foam rolling volumes in the interset rest period on maximum repetition performance. Hong Kong Physiotherapy Journal, 36 (1), 57-62.

Monteiro, E. R.; Wakefield, B.; Ribeiro, M. S.; Fiuza, A. G.; Cunha, J. C. O. W.; Novaes, G. S.; Panza, P. S.; Vianna, J. M.; Novaes, J. S. (2018). Automassagem e alongamento nas regiões anterior e posterior de coxa aumentam de forma aguda a amplitude articular de ombro. Motricidade, 14 (1), 174182.

Monteiro, E. R.; Costa, P. B.; Corrêa Neto, V. G.; Hoogenboom, B. J.; Steele, J.; Silva Novaes, J. D. (2019). Posterior thigh foam rolling increases Knee extension fatigue and passive shoulder range-of-motion. J Strength Cond Res, 33 (4), 987-994.

Myers, T. W. Trilhos anatômicos. 3. ed. São Paulo: Manole pp. 376; 2016.

Rodrigues, M. E.; Marins, J. C. B. (2011). Counter movement e squat jump: análise metodológica e dados normativos em atletas. $R$ Bras Ci e Mov, 19 (4), $108-119$.

Schleip, R. (2003). Fascial plasticity - a new neurobiological explanation: Part 1. J Bodyw Mov Ther, 7 (2), $575-580$.

Stecco, C.; Schleip, R. (2016). A fascia and the fascial system. J Bodyw Mov Ther, 20 (1), 139-140.

Toledo, L.; Bentes, C. M.; Miranda, H.; Palma, A.; Corrêa Neto, V. G. (2014). Efeito agudo do alongamento estático sobre a potência muscular em atletas de futebol feminino sub-17. Conscientiae Saúde, 13 (2), 272-280.

Zakas, A.; Doganis, G.; Galazoulas, C.; Vamvakoudis, E. (2006). Effect of acute static stretching duration on isokinetic peak torque in pubescent soccer player. Pediatric Exercise Science, 18 (2), 252-261.

Zhang, Q.; Trama, R.; Fouré, A.; Hautier, C. A. (2020). The immediate effect of self-myofascial release on flexibility, jump performance and dynamic balance ability. J Hum Kinet, 75, 139-148. 